\title{
Tellurium as a successor of silicon for extremely scaled nanowires: a first-principles study
}

\author{
Aaron Kramer ${ }^{1}$, Maarten L. Van de Put ${ }^{2}$, Christopher L. Hinkle ${ }^{3}$ and William G. Vandenberghe ${ }^{2 凶}$
}

Trigonal-Tellurium (t-Te) has recently garnered interest in the nanoelectronics community because of its measured high hole mobility and low-temperature growth. However, a drawback of tellurium is its small bulk bandgap ( $0.33 \mathrm{eV})$, giving rise to large leakage currents in transistor prototypes. We analyze the increase of the electronic bandgap due to quantum confinement and compare the relative stability of various $t-T e$ nanostructures ( $\mathrm{t}$-Te nanowires and layers of $\mathrm{t}$-Te) using first-principles simulations. We found that small t-Te nanowires $\left(\leq 4 \mathrm{~nm}^{2}\right)$ and few-layer t-Te ( $\leq 3$ layers) have bandgaps exceeding $1 \mathrm{eV}$, making Tellurium a very suitable channel material for extremely scaled transistors, a regime where comparably sized silicon has a bandgap that exceeds $4 \mathrm{eV}$. Through investigations of structural stability, we found that t-Te nanowires preferentially form instead of layers of $t$-Te since nanowires have a greater number of van der Waals $(\mathrm{vdW})$ interactions between the t-Te-helices. We develop a simplified picture of structural stability relying only on the number of vdW interactions, enabling the prediction of the formation energy of any $\mathrm{t}$-Te nanostructure. Our analysis shows that $\mathrm{t}$-Te has distinct advantages over silicon in extremely scaled nanowire transistors in terms of bandgap and the t-Te vdW bonds form a natural nanowire termination, avoiding issues with passivation.

npj 2D Materials and Applications (2020)4:10; https://doi.org/10.1038/s41699-020-0143-1

\section{INTRODUCTION}

With the continued scaling of transistor size, the silicon (Si) mobility reduces drastically ${ }^{\uparrow}$ and at extremely small dimensions, the silicon bandgap increases dramatically (see Fig. 1). Reduced mobility gives rise to a reduced on-current, while a bandgap that is too large reduces the relative height of the gate dielectric potential barrier, increasing gate leakage current ${ }^{2}$. An increased bandgap may also lead to severe challenges in terms of doping and contacting. Graphene and topological insulators present a solution to the low mobility problem, but unfortunately have a vanishing bandgap, making the realization of a low off-state current challenging ${ }^{3-5}$. Several other alternative materials with a bandgap have been proposed, but no single material has emerged that can clearly outperform $\mathrm{Si}$ at the nanoscale ${ }^{6}$. Transition-metal dichalcogenides demonstrate a mobility that is lower and a bandgap that is higher than desired ${ }^{7,8}$. Phosphorene showed initial promise, but theoretical studies reveal a severely degraded mobility at small dimensions ${ }^{9}$.

Trigonal-Tellurium ( $\mathrm{t}-\mathrm{Te}$ ) is an alternative material of interest because a high hole mobility $(707 \mathrm{~cm} / \mathrm{V} / \mathrm{s})$, low-temperature growth $\left(\leq 120^{\circ} \mathrm{C}\right)^{10}$, and a high current density $\left(1 \mathrm{~A} / \mathrm{mm}^{2}\right)$ have all been demonstrated ${ }^{11}$. $\mathrm{t}$-Te has a nearly direct and small bulk bandgap $(0.33 \mathrm{eV})^{12}$, which has historically enabled applications in thermoelectric $^{13}$, piezoelectric ${ }^{14}$, and photoconductive devices ${ }^{15}$, as well as infrared detectors ${ }^{16}$. For transistor applications, the small bandgap is detrimental, but, at scaled dimensions, quantum confinement effects are expected to increase the bandgap significantly (see Fig. 1). A significant increase in bandgap eliminates the major drawback of Te as a channel material for extremely scaled future transistors. Moreover, there has recently been an experimental demonstration of Te nanowires with a diameter down to $2 \mathrm{~nm}$ through the encapsulation in carbon nanotubes ${ }^{17}$.
Structurally, t-Te comprises one-dimensional (1D) helical chains of covalently bonded Te atoms (primary interaction). These Tehelices form a trigonal lattice through a mixture of covalent bonding and van der Waals (vdW) interactions (secondary interaction). The existence of the primary and secondary interactions and the overall helical t-Te structure are a consequence of Te comprising of six valence electrons ${ }^{18}$. Analogous to layered vdW materials, such as graphene ${ }^{19-21}$ and transition-metal dichacogenides $^{7,8,22}$, the vdW interactions between neighboring t-Tehelices readily yield two-dimensional (2D) and 1D nanostructures by exfoliation or a well-controlled growth.

Several low-dimensional phases of $\mathrm{Te}$, such as monolayer Te, named Tellurene, have been investigated using first-principles density functional theory (DFT). A rich landscape of predicted phases has been uncovered by Zhu et al. ${ }^{23}(\alpha, \beta$, and $\gamma)$, Liu et al. ${ }^{24}$ $(\delta$ and $\eta)$, and Xian et al. ${ }^{25}$ (square Tellurene), where only the $\beta$ phase monolayer resembles bulk t-Te. The $a, \delta$, and $\eta$ phases were predicted to be more stable than the $\beta$-phase, with the $\eta$-phase being the most stable ${ }^{23,24}$. However, these alternative phases remain elusive since only layers of the bulk-like $\beta$-phase have been successfully grown experimentally and used in field-effect transistors (FETs) so far ${ }^{10,11,26}$. Unfortunately, little is known about the relative stability of the bulk-like " $\beta$-phase" layers of Te compared to t-Te nanowires or what bandgaps are anticipated for tellurium nanostructures.

In this paper, we employ first-principles calculations within the DFT framework to compare and analyze the stability of various ideal pristine cases of $\mathrm{t}$-Te nanostructures (nanowires and layers). We determine the bandgaps of t-Te nanowires and find that thin nanowires $\left(<4 \mathrm{~nm}^{2}\right)$ and few layers ( $\leq 3$ layers) of Te have bandgaps exceeding $1 \mathrm{eV}$, making t-Te a suitable channel material for extremely scaled FETs. We calculate formation energies, study the surface-tovolume ratios, and develop a simplified model to determine the

\footnotetext{
${ }^{1}$ Department of Physics, University of Texas at Dallas, 800 W. Campbell Road, Richardson, TX 75080, USA. ${ }^{2}$ Department of Materials Science and Engineering, University of Texas

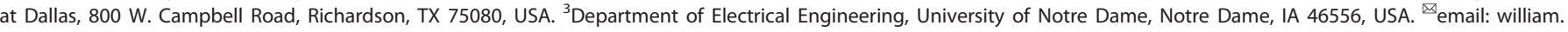
vandenberghe@utdallas.edu
} 

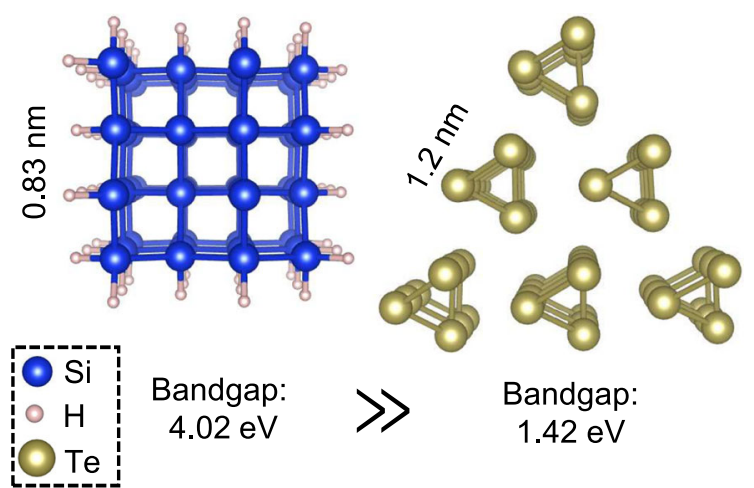

Bandgap: $4.02 \mathrm{eV}$ )

Bandgap: $1.42 \mathrm{eV}$

Fig. 1 Comparison of a $4 \times 4$ Si nanowire $(0.83 \mathrm{~nm}$ side length) with a Te nanowire $(1.2 \mathrm{~nm}$ side length). Both nanowires have comparable cross-sectional areas. We compute the bandgap of both structures as outlined in the "Methods" section. The silicon bandgap, exceeding $4 \mathrm{eV}$, will result in major challenges for transistor applications such as reduced mobility and contacting difficulty. A similar size tellurium nanowire has a well-suited $1.42 \mathrm{eV}$ bandgap.
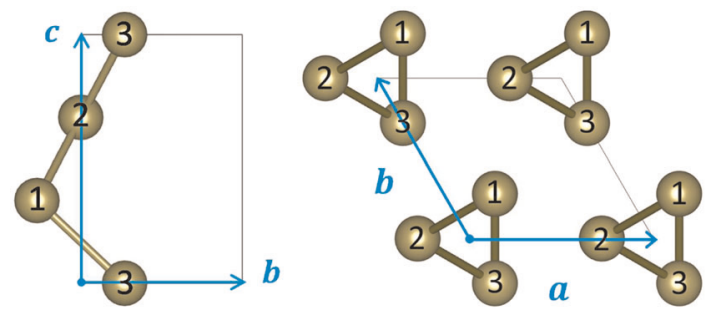

Fig. 2 Structure of bulk t-Te (three atoms per unit cell). Spheres and lines represent Te atoms and covalent bonds within the Tehelix. The blue arrows illustrate the three primitive axes of the crystal.

stability of 1D nanostructures using an energy penalty $\epsilon_{m}$ of a Te-helix with $m$ missing helical neighbors. We find that 1D hexagonal-shaped $\mathrm{t}$-Te nanowires are thermodynamically the most favored. This stability is explained using our simplified model.

\section{RESULTS AND DISCUSSION}

Atomic structure

Figure 2 illustrates the bulk t-Te structure. The symmetries of bulk tTe have been well studied, with t-Te belonging to point group $D_{3}$ and crystalizing into right-handed helices (space group $P 3_{3} 21$ ) or left-handed helices (space group $P 3_{2} 21$ ) $^{27-30}$. For this paper, we will consider the right-handed variant only, but the identical results would be obtained for the left-handed variant. The six symmetry operations associated with the space group are: the identity, two screw axes along the $c$ direction, a $C_{2}$ rotation about axis $a$ or $b$, and the combinations of each screw axis with the $C_{2}$ rotation.

Figure 3 illustrates all $73 \mathrm{t}$-Te nanostructures under investigation in this paper. We study $16 \mathrm{t}$-Te nanowires (triangular, rhomboid, and hexagonal), $48 \beta$-phase Te nanoribbons (monolayer, bilayer, and trilayer), and nine sheets of $\beta$-phase Te. The nanowires are labeled by the number of helices on a side $(N)$ that translates into a total of $N(N+1) / 2$ helices for triangular, $N^{2}$ helices for rhomboid, and $3 N(N-1)+1$ helices for hexagonal nanowires. Nanoribbons are sheets of Te identified by a thickness of $L$ Te-helices and a width of $R$ Te-helices, for a total of $L R$ Te-helices. We study all aforementioned ribbon/nanowire configurations containing up to 37 helices (111 atoms) in a unit cell.

Most nanostructures have fewer symmetry operations compared to bulk t-Te. Hexagonal nanowires (Fig. 3c) are the exception and maintain all symmetry operations. Rhomboid nanowires (Fig. 3b) only maintain a $C_{2}$ rotation about axis $a$, while triangular nanowires (Fig. 3a) only maintain the two screw axes. All structures maintain the vdW-linked helix structure, except for the monolayer sheet (Fig. $3 g, L=1)$. In the monolayer, helices are covalently bonded instead ${ }^{23,31-33}$.

We calculate the bulk t-Te lattice constants as $a_{\text {bulk }}=4.40 \AA$ and $c_{\text {bulk }}=5.93 \AA$, and the bulk intra-helix bond length as $d_{\text {bulk }}=2.90 \AA$, which agrees with previous calculations ${ }^{23,34}$. The experimental bulk t-Te lattice constants are $a_{\operatorname{Exp}}=4.45 \AA$ and $c_{\text {Exp }}=5.93 \AA^{35}$.

Table 1 shows the calculated lattice constants for the multi-layer sheets of Te, $a_{2 D}$ and $c$, and the bond lengths $d$. The lattice constants and bond lengths quickly reach the bulk values with increasing sheet thickness with a deviation within $1 \%$ for three layers or more. In a monolayer sheet, that is, Te, neighboring helices are bound more tightly than in all other configurations. Tellurene has a secondary bond length of only $3.03 \AA$ between nearest atoms in neighboring helices.

Table 2 shows the lattice constant along the helix axis (c), the quasi-lattice constants $\tilde{a}$ (defined in the Methods section), and the intra-helix covalent bond lengths $d$ for the various t-Te nanowires. For this case, the quasi-lattice constants are also within $1 \%$ of the bulk lattice constant, although they do not exactly approach the bulk values for the sizes we have simulated.

The lattice constants $a_{2 \mathrm{D}}$ (Table 1), the quasi-lattice constants $\tilde{a}$ (Table 2), and the bulk t-Te lattice constant ( $4.40 \AA)$ all show: $\tilde{a}>a_{\text {bulk }} \geq a_{2 \mathrm{D}}$. t-Te nanowires and sheets of Te have a smaller lattice constant $c$ and intra-helix bond length $d$ than their respective bulk t-Te values.

\section{Electronic bandstructure}

In Fig. 4, we show the conduction and valence band offsets, computed using a hybrid DFT scheme, including spin-orbit coupling (see Methods section), for all but our largest nanowires and many sheets of Te, where the computational burden becomes excessive. As explained in the "Methods" section, we use a hybrid DFT scheme that is known to vastly improve over standard DFT techniques for bandgap predictions. For example, the bulk $\mathrm{Si}$ bandgap is severely underestimated using standard DFT to be $0.6 \mathrm{eV}$, while the hybrid DFT technique we use predicts a much more accurate $1.2 \mathrm{eV}$ bandgap (1.12 eV experiment) ${ }^{36}$. For bulk tTe we find a hybrid DFT $0.26 \mathrm{eV}$ bandgap, which is close to the experimentally measured $0.33 \mathrm{eV}^{10}$, validating our use of hybrid DFT for Te. We found that the calculation for a single Te-helix in ref. ${ }^{17}$ does not employ hybrid functionals and underestimates the bandgap to be $1.51 \mathrm{eV}$ compared to the $2.2 \mathrm{eV}$ we find.

In Fig. 4a, a significant increase in bandgap is observed as quantum confinement effects become more pronounced for smaller nanowires. For the t-Te nanowires, the bandgaps range from 0.8 to $2.19 \mathrm{eV}$. For the Si nanowires, the bandgaps range from 1.7 to $4.0 \mathrm{eV}$. For sheets of Te, the bandgaps range from 0.64 to $1.43 \mathrm{eV}$. Comparing t-Te nanowires with the same number of Tehelices, the order of largest to smallest bandgaps proceeds from triangular, to rhomboid, to hexagonal nanowires.

Figure 5 shows the calculated bandstructures for monolayer and bilayer Te sheets and three t-Te nanowires $(N=2$ hexagonal, $N=3$ rhomboid, and $N=4$ triangular) using the hybrid DFT scheme. Figure $5 \mathrm{a}$ shows that monolayer Te has a direct gap of $1.4 \mathrm{eV}$ (located at $\Gamma$ ) and bilayer Te has an indirect bandgap of $1.2 \mathrm{eV}$. Figure $5 \mathrm{~b}, \mathrm{~d}$, and e shows that all three t-Te nanowires are indirect bandgap materials with bandgap values $0.98 \mathrm{eV}$ (hexagonal), $1.1 \mathrm{eV}$ (rhomboid), and $1.2 \mathrm{eV}$ (triangular). Since bulk t-Te has a nearly direct bandgap, we expect nanowires with areas in excess of $6 \mathrm{~nm}^{2}$ to have nearly direct bandgaps. As a comparison, all Si nanowires are direct bandgap (located at Г) materials.

To explain the different size scaling behavior of the electronic bandgap in $\mathrm{t}-\mathrm{Te}$, compared to a bulk material such as $\mathrm{Si}$, we show 
(a) Triangular Te nanowires

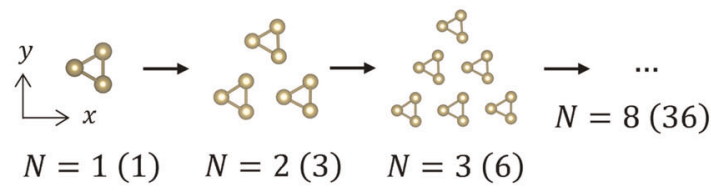

(b) Rhomboid Te nanowires

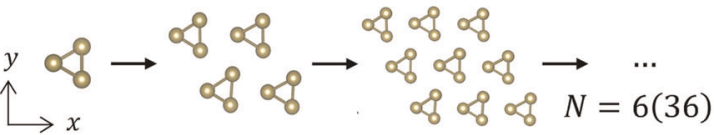

$$
\begin{aligned}
& N=1(1) \quad N=2(4) \quad N=3(9)
\end{aligned}
$$

(d) Monolayer Tellurene Nanoribbons: $L=1$

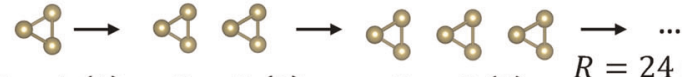

$$
\begin{aligned}
& y \quad R=1(1) \quad R=2(2) \quad R=3 \text { (3) } \\
& \longleftrightarrow x
\end{aligned}
$$

(e) Bilayer Tellurene Nanoribbons: $L=2$

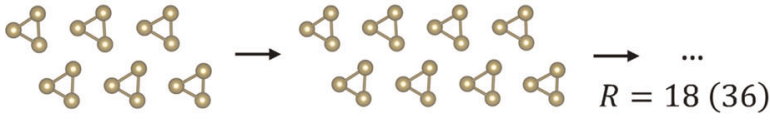

$$
\begin{aligned}
& R=4 \text { (8) }
\end{aligned}
$$

(c) Hexagonal Te nanowires

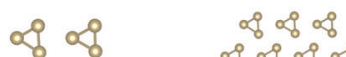

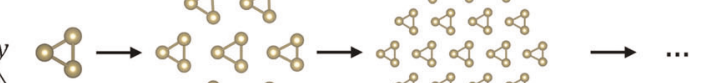

$$
\begin{aligned}
& \longrightarrow x \quad \text { of of } \quad \text { of of of of } \quad N=4 \text { (37) } \\
& N=1(1) \quad N=2(7) \quad N=3(19)
\end{aligned}
$$

(f) Trilayer Tellurene Nanoribbons: $L=3$

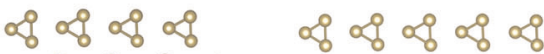

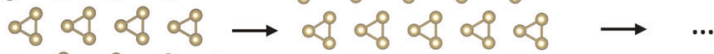

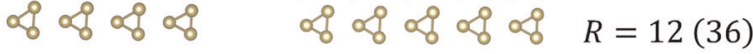

$$
\begin{aligned}
& \begin{array}{ll}
y & R=4(12)
\end{array} \\
& R=5 \text { (15) }
\end{aligned}
$$

(g) Tellurene Sheets
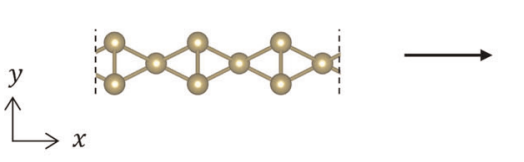

$$
\begin{aligned}
& \text { \% \% \% \% } \\
& \text { \% \& \% \% }
\end{aligned}
$$

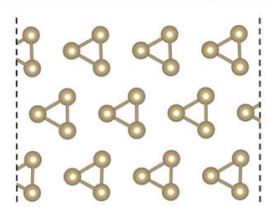

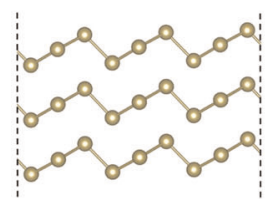

$$
L=3
$$<smiles>COOOOOOOOO</smiles>

$$
L=1
$$
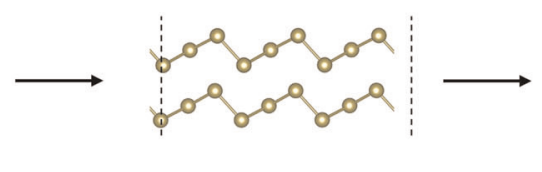

$L=2$

Fig. 3 Cross-sectional view of Tellurium nanostructures. a triangular, $\mathbf{b}$ rhomboid, $\mathbf{c}$ hexagonal nanowires, and Tellurene $\mathbf{d}$ monolayer, e bilayer, and $\mathbf{f}$ trilayer ribbons viewed from the [001] direction. The $\mathbf{g}$ Tellurene sheets are viewed from the [001] and [100] directions. For Te nanowires, $N$ is the number of Te-helices per nanowire side. For Tellurene nanoribbons, $R$ is the number of Te-helices on the longer side (direction of increasing nanoribbon size). The numbers within parentheses are the total number of Te-helices. For the Tellurene sheets, $L$ is the number of Tellurene sheet layers.

the dependence of the bandgap on the average number of helical neighbors per Te-helix $\bar{m}$ in Fig. 6 . We find that the bandgap linearly depends on $\bar{m}$. Interestingly, this linear behavior also includes the edge cases of bulk and a single t-Te strand. This behavior agrees with previous findings in Selenene (2D monolayer Selenium) in ref. ${ }^{37}$, where the authors highlight the dependence of the bandgap on the overlap of the bonding (valence band) and anti-bonding (conduction band) orbitals between adjacent Se helices rather than pure quantum confinement effects. Similarly, for $\mathrm{t}-\mathrm{Te}$, we see that increasing the number of interactions (overlaps) between neighboring Te-helices decreases the bandgap linearly. We note that, compared to Se, the Te bandgaps are smaller due to Te's stronger spin-orbit coupling (higher atomic mass).

\section{Formation energy}

Figure 7 shows the formation energies for all nanoribbons and tTe nanowires. The nanowires have a lower formation energy compared to the nanoribbons for the same number of Te-helices (or total cross-sectional area). Hexagonal nanowires have the lowest formation energy. Rhomboid and triangular nanowires

\begin{tabular}{|lccc|}
\hline Table 1. & Lattice constants of multi-layer sheets of Tellurene. & \\
\hline Name & $a_{2 \mathrm{D}}(\AA)$ & $c(\AA)$ & $d(\AA)$ \\
\hline 1-Layer & 4.22 & 5.61 & 2.77 \\
& & 5.79 & $3.03^{\mathrm{a}}$ \\
2-Layer & 4.33 & 5.84 & 2.87 \\
3-Layer & 4.36 & 5.87 & 2.87 \\
4-Layer & 4.38 & 5.88 & 2.88 \\
5-Layer & 4.39 & 5.89 & 2.88 \\
6-Layer & 4.39 & 5.90 & 2.89 \\
7-Layer & 4.40 & 5.91 & 2.89 \\
8-Layer & 4.40 & 5.91 & 2.89 \\
9-Layer & 4.40 & 2.89 \\
\hline Lattice constants $a_{2 D}$ and $c$ and intra-helix bond lengths $d$ for multi-layer \\
sheets of Tellurene. \\
a 1-Layer has a second bond length.
\end{tabular}

have the second and third lowest formation energies, respectively. The monolayer ribbons have an exceedingly high formation energy, while the bilayer and trilayer ribbons have formation energies closer, but still higher, than the nanowires. 
The exceedingly high formation energy of monolayer $\mathrm{Te}$ indicates that the experimental realization of monolayer $\mathrm{Te}$ without substrate support will be very unlikely. Interestingly, the formation energy cost is cut significantly for the bilayers and trilayers. We attribute this to the different fundamental structure of monolayer Te, shown in Fig. 3g. None of the known monolayer $\mathrm{t}$-Te phases have clearly separated helices. The bilayer $(\mathrm{L}=2)$ and trilayer $(L=3)$ do have distinguishable helices as illustrated in Fig. $3 g$. Interestingly, experimental growth also found that wires rather than layers are preferred ${ }^{10}$, which agrees with our findings.

Table 2. Nanowire quasi-lattice constants.

\begin{tabular}{lrccc}
\hline Name & $n$ & $\tilde{a}(\AA)$ & $c(\AA)$ & $d(\AA)$ \\
\hline Single Hex & 1 & - & 5.69 & 2.75 \\
$N=2 \mathrm{Hex}$ & 7 & 4.46 & 5.79 & 2.83 \\
$N=3 \mathrm{Hex}$ & 19 & 4.44 & 5.84 & 2.86 \\
$N=4 \mathrm{Hex}$ & 37 & 4.43 & 5.87 & 2.87 \\
$N=2 \mathrm{Rhm}$ & 4 & 4.44 & 5.75 & 2.81 \\
$N=3 \mathrm{Rhm}$ & 9 & 4.44 & 5.79 & 2.84 \\
$N=4 \mathrm{Rhm}$ & 16 & 4.44 & 5.82 & 2.85 \\
$N=5$ Rhm & 25 & 4.44 & 5.84 & 2.86 \\
$N=6$ Rhm & 36 & 4.43 & 5.86 & 2.87 \\
$N=2$ Tri & 3 & 4.41 & 5.72 & 2.80 \\
$N=3$ Tri & 6 & 4.43 & 5.75 & 2.83 \\
$N=4$ Tri & 10 & 4.43 & 5.78 & 2.84 \\
$N=5$ Tri & 15 & 4.43 & 5.81 & 2.85 \\
$N=6$ Tri & 21 & 4.43 & 5.83 & 2.86 \\
$N=7$ Tri & 28 & 4.43 & 5.84 & 2.87 \\
$N=8$ Tri & 36 & 4.43 & 5.85 & 2.87 \\
\hline Quasi-lattice constant $\tilde{\boldsymbol{a}}$, lattice constant $\boldsymbol{c}$, and intra-helix bond $\boldsymbol{d}$ for all Te \\
nanowires. $\boldsymbol{n}$ is the number of Te-helices. Hex, Rhm, and Tri are short for \\
hexagonal, rhomboid, and triangular Te nanowires, respectively.
\end{tabular}

To understand the differences in the formation energies among the remaining nanostructures, we compute the surface-to-volume ratio for all $\mathrm{t}$-Te nanowires and the bilayer and trilayer nanoribbons. The computed surface-to-volume ratios are shown in Fig. 8. Hexagonal nanowires have the lowest surface-to-volume ratio for the same number of Te-helices. This agrees with previous results in Fig. 7 where hexagonal nanowires have the lowest formation energy.

Unfortunately, surface-to-volume ratio does not explain all observed differences. For instance, bilayer and trilayer nanoribbons have a lower surface-to-volume ratio compared to the triangular nanowires when the nanoribbons have more than 14 and 27 helices, respectively. To explain the observation in Fig. 7, that nanowires always exhibit a lower formation energy compared to nanoribbons, we need an analysis going beyond simple surface-to-volume ratio considerations.

To this end, we propose an alternative model, based on the observation that nanostructures where Te-helices with more neighboring Te-helices have lower formation energies. In particular, Te-helices are "happy" when they have six helical neighbors, in which case their local environment resembles bulk t-Te. Nanowires will always have a higher ratio of "happy" helices to the total number of helices than Te nanoribbons.

To determine whether the number of neighboring helices is a good metric, we extract an energy penalty $\epsilon_{m}$ for a helix with a given number $(m \leq 6)$ of missing neighboring helices. The energy penalty $\epsilon_{m}$ is given per unit cell. When all helices are missing, $m=6$ and the energy penalty is the formation energy of a single helix unit cell. For $m \in(0,2,3,4)$, the penalties are determined using an ordinary least-squares (OLS) fit on all the tTe nanowires and nanoribbons under study, except for the structurally different monolayers. No structures were considered that contain a helix with $m=1$ missing helical neighbors.

Figure 9 shows the resulting energy penalties per unit cell. Significant energy penalties of 0.80 to $0.40 \mathrm{eV}$ are observed for helices with four to two missing helical neighbors. The maximum energy penalty for a helix without neighbors is $1.17 \mathrm{eV}$. The small value $\epsilon_{0}=0.01 \mathrm{eV}$ indicates that the OLS almost exactly reproduces the limit of bulk t-Te. Furthermore,

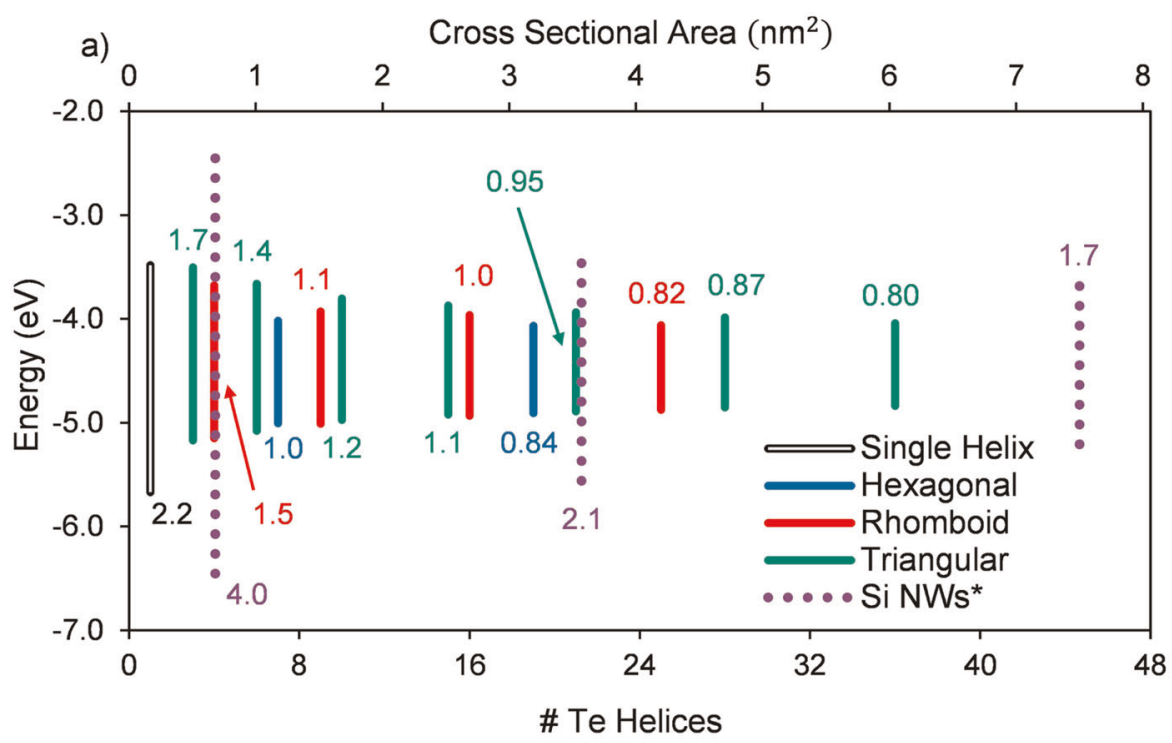

b)

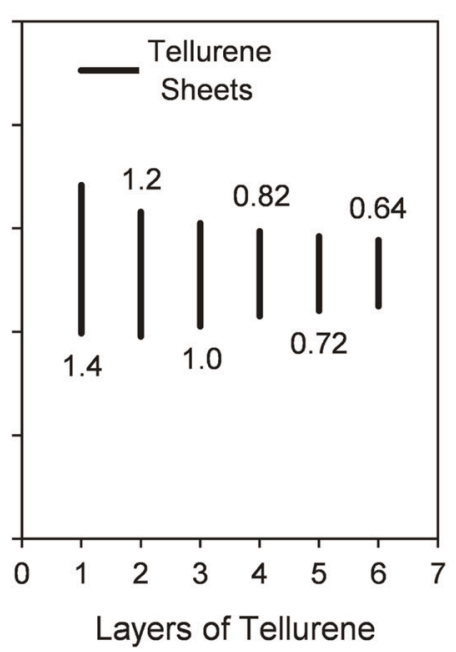

Fig. 4 Band alignment of tellurium nanostructures and silicon nanowires. a Conduction and valence band offsets of Te (using SOC+HSE06) and Si (using HSE06) nanowires as a function of the number of Te-helices or cross-sectional area. $\mathbf{b}$ Conduction and valence band offsets of sheets of Tellurene as a function of number of Tellurene layers (using SOC+HSE06). The annotation attached to each line is the bandgap of each material. The vacuum level is set to $0 \mathrm{eV}$. ${ }^{*}$ The silicon nanowires (NWs) are only a function of total cross-sectional area (top axis). 
(a) Sheets of Tellurene

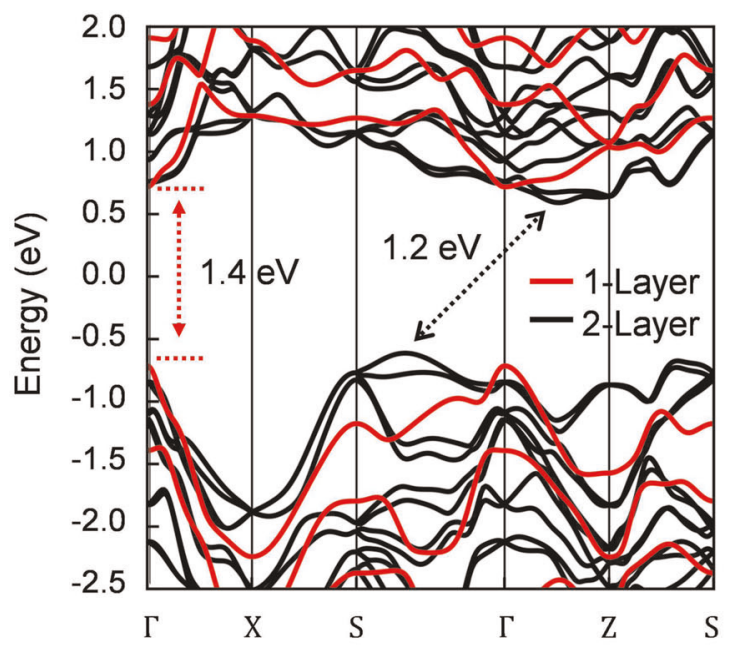

(b) $\mathrm{N}=2$ Hexagonal

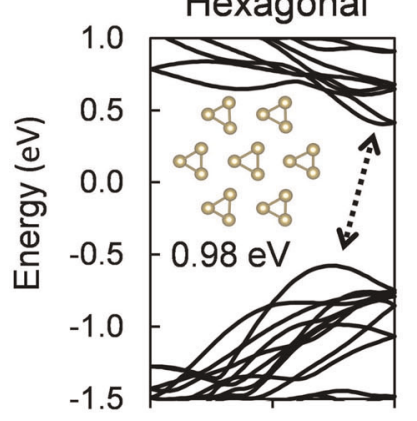

(c)

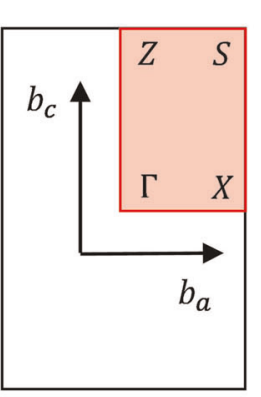

(d) $\quad \mathrm{N}=3$ Rhomboid

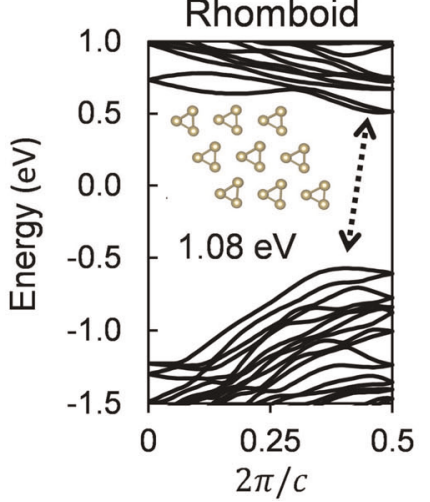

(e) $\quad \mathrm{N}=4$
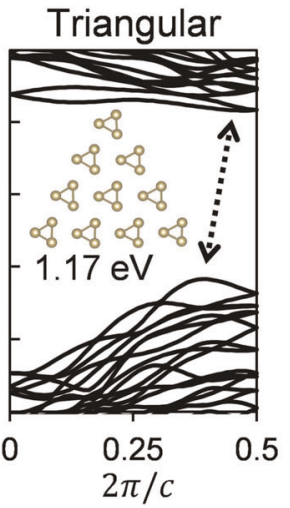

Fig. 5 Bandstructures of Tellurene sheets. a monolayer and bilayer sheets of Tellurene and the $\mathbf{b} N=2$ hexagonal nanowire bandstructures. The c first Brilliouin zone for sheets of Tellurene and the calculated bandstructures for $\mathbf{d} N=3$ rhomboid and e $N=4$ triangular nanowires. The Fermi level is at 0eV. The arrows on the bandstructures indicate the conduction band minima and valence band maxima.

the energy penalties show remarkable linearity, where, on average, the energy penalty of removing a neighboring helix is $\sim 0.20 \mathrm{eV}$. This linear scaling behavior in the energy penalty can be applied to similar trigonal vdW materials such as Selenium.

By applying these energy penalties to the nanostructures under consideration, we reproduce the order of largest to lowest

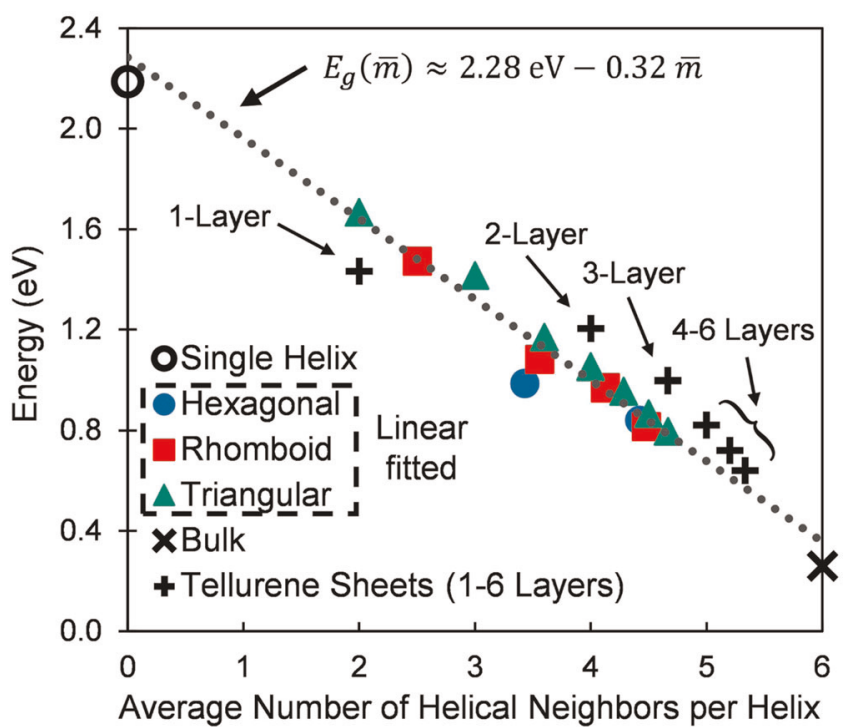

Fig. 6 Electronic bandgaps $\left(E_{\mathrm{g}}\right)$ of all t-Te nanowires and sheets of Tellurene with the average number of helical neighbors per helix $\bar{m}$, calculated with HSEO6 functionals, including spin-orbit interaction. The dotted lines represent a linear fit on the hexagonal, rhomboid, and triangular t-Te nanowires. We extrapolate the linear fit to cover the single helix, and the bulk t-Te domains, showing excellent agreement.

formation energies, shown in Fig. 7. This demonstrates that the energy penalty picture (with a penalty due to missing neighboring helices), in contrast to the surface-to-volume ratio picture, captures correctly that $\mathrm{t}$-Te nanowires have a lower formation energy compared to nanoribbon-like nanowires. The energy penalty picture also explains why hexagonal- and triangularshaped nanowires have the lowest and highest formation energies among the nanowires. Therefore, the number of helical neighbors is a better metric for formation energy than surface-tovolume ratio in $1 \mathrm{D}$ vdW structures.

Considering experimental growth, we predict that growth of hexagonal nanowires will be favored. However, it is possible to envision that a surface interaction modifies the surface energy and makes surface helices "happier." If this is the case, growth of nanowires with more missing helices can be anticipated. In the presence of such a favorable surface interaction, we expect triangular nanowires to form preferentially. This agrees with experiments where hexagonal- and triangular-shaped nanowires form under various conditions, while rhomboid-shaped nanowires are never observed ${ }^{38}$

\section{METHODS}

First-principles calculations

We perform first-principles calculations with DFT as implemented in the Vienna Ab-initio Software Package (VASP) with projector augmented waves, a generalized gradient approximation using the exchangecorrelation functional from Perdew-Burke-Ernzerhof $(\mathrm{PBE})^{39,40}$, the vdW correction (DFT-D3) from Grimme, Ehrlich, and Krieg ${ }^{41}$, and a kinetic cutoff of $200 \mathrm{eV}$ for the plane-wave basis set. For charge density calculations, the $\mathrm{t}$-Te nanowires, the $\mathrm{t}-\mathrm{Te}$ nanoribbons, the sheets of $\mathrm{Te}$, and bulk $\mathrm{t}-\mathrm{Te}$ calculations were performed using a $1 \times 1 \times 4,1 \times 1 \times 4,6 \times 1 \times 4$, and $6 \times$ $6 \times 4$ Monkhorst-Pack $k$-point sampling, respectively ${ }^{42}$. Several stu$\operatorname{dies}^{23,24,31,34}$ indicate that there is "no a priori knowledge" in choosing the correct van der Waals correction. Based on a small-scale study, we found that the lattice constants change by $<2 \%$ when using a different van der Waals correction (vdb-optB88 ${ }^{43}$ ). 
(a)

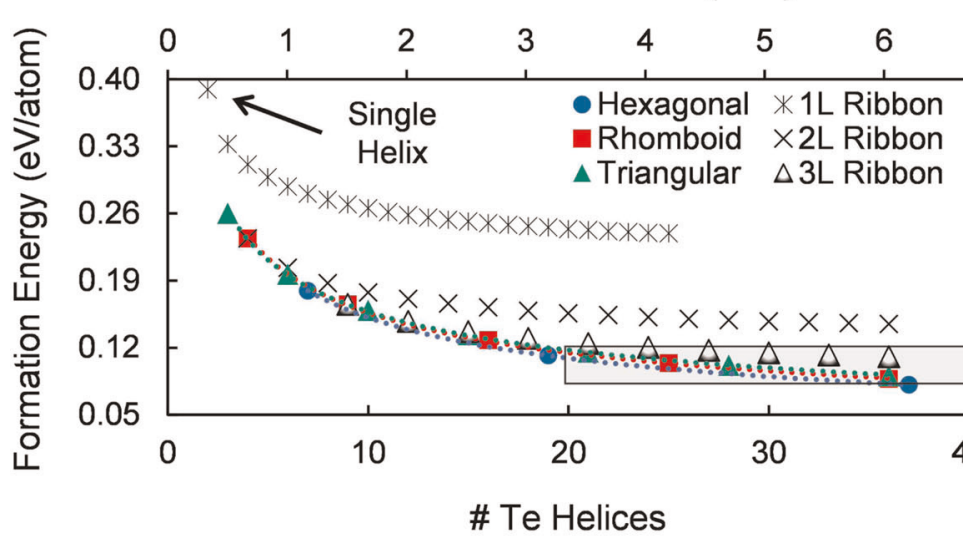

(b) Cross Sectional Area $\left(\mathrm{nm}^{2}\right)$

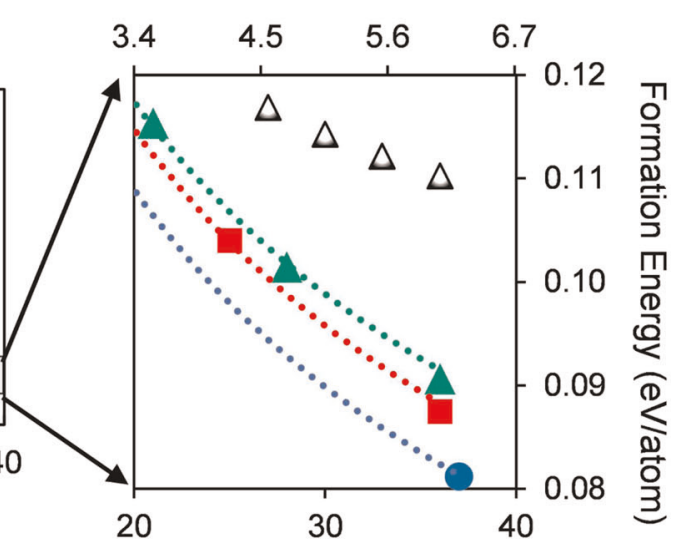

\# Te Helices

Fig. 7 Formation energy analysis. a Formation energies of all t-Te nanowires and Tellurene nanoribbons and $\mathbf{b}$ zoomed in formation energies as a function of total number of Te-helices or total cross-sectional area. $1 \mathrm{~L}, 2 \mathrm{~L}$, and $3 \mathrm{~L}$ stand for monolayer, bilayer, and trilayer nanoribbons, respectively. The dotted lines are power fits to guide the eye.

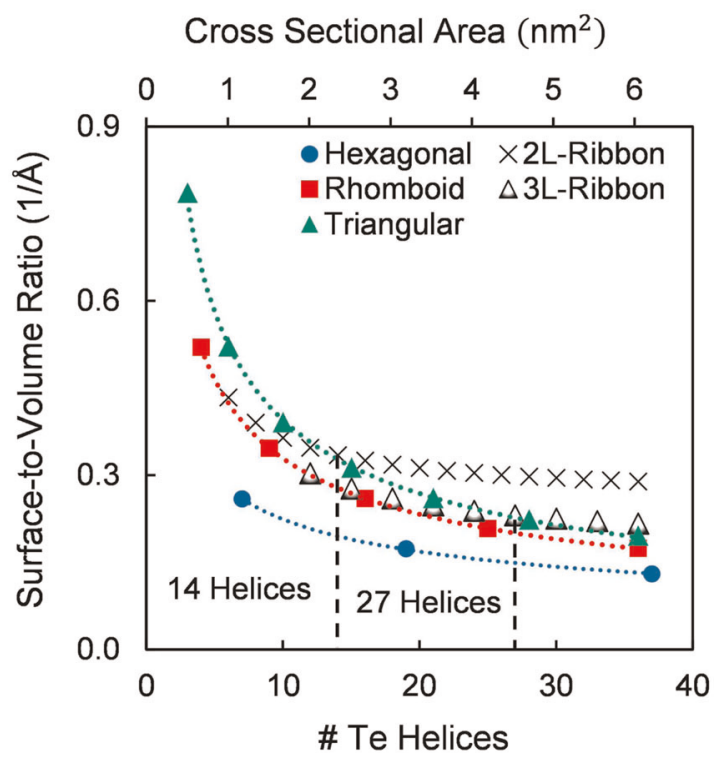

Fig. 8 Surface-to-volume ratio for the t-Te nanowires and the nanoribbons as a function of the number of Te-helices or total cross-sectional area. Solid points are calculated values and dotted lines are power fits to guide the eye. $2 \mathrm{~L}$ and $3 \mathrm{~L}$ stand for bilayer and trilayer, respectively.

Calculations on bulk Si and all Si nanowires were performed with a $300 \mathrm{eV}$ kinetic cutoff with Monkhorst-Pack $k$-point charge density sampling of $4 \times 4 \times 4$ and $1 \times 1 \times 4$, respectively.

To create t-Te nanowires, nanoribbons, and sheets of $\mathrm{Te}$, we first construct supercells from the bulk t-Te atomic coordinates and lattice parameters. Next, we remove excess atoms, and pad with $10 \AA$ of surrounding vacuum. We relax all structures until all forces are no $<5 \mathrm{meV} /$ A. All relaxations use the PBE + DFT-D3 scheme.

The hybrid DFT schemes used HSE06 functionals for the conduction and valence band offsets ${ }^{44}$. We incorporate spin-orbit coupling for all conduction and valence band offsets, and bandstructure calculations as implemented in VASP ${ }^{45}$.

The formation energy is $E_{\mathrm{F}}=E_{\text {tot }} / N_{\text {tot }}-\epsilon_{\text {bulk. }}$. Where $E_{\text {tot }}$ is the total ground state energy of a nanostructure, $N_{\text {tot }}$ is the total number of atoms per supercell of a nanostructure, and $\epsilon_{\text {bulk }}$ is the cohesive energy of bulk t$\mathrm{Te}$, which we calculate as $-3.40 \mathrm{eV} /$ atom.

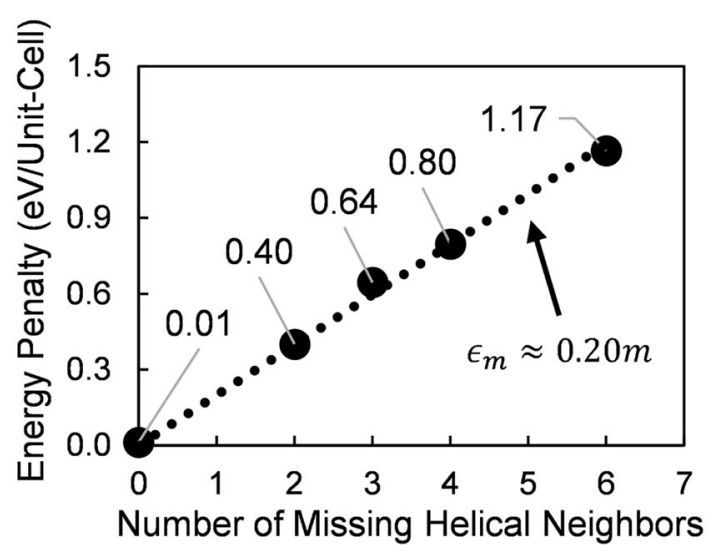

Fig. 9 The energy penalties as a function of the number of missing helical neighbors $\mathbf{m}$. Solid circles are OLS fit on the formation energies of all structures, except for the monolayers. The dotted line is a linear fit (equation shown on graph) through the origin. The energy penalty is relative to bulk t-Te.

Lattice constants and surface-to-volume ratio

To calculate the surface-to-volume ratio $(r)$, we use the lattice constant in the periodic direction $(c)$ in addition to a "quasi-lattice constant" $(\tilde{a})$ in the non-periodic directions.

The calculation of the quasi-lattice constant proceeds as follows: We select the three planes, perpendicular to the $z$-axis (crystal axis c) containing the Te atoms. Within each plane, we calculate the sum, and then average out all the nearest-neighbor distances $\ell_{p, i}$ for each atom in the plane, where $i$ denotes the nearest-neighbor atom and $p$ denotes a plane. As a closed-form equation, the quasi-lattice constant is given by the average over the three planes:

$\tilde{a}=\frac{1}{3 N_{i}} \sum_{p=1}^{3} \sum_{i=1}^{N_{i}} \ell_{p, i}$,

where $N_{i}$ equals the total number of nearest-neighbor distances per plane. This formula averages distances across all three planes. If the entire structure retains the screw axes of bulk $\mathrm{t}-\mathrm{Te}$, then the averaging distances across one plane is sufficient. Figure 10 illustrates the methodology for the $N=3$ rhomboid t-Te nanowire.

The surface-to-volume ratio for $\mathrm{t}$-Te nanowires and nanoribbons is $r=A_{\llcorner} / V$, where $A_{\llcorner}$is the lateral surface area and $V$ is volume (per supercell). Nanowire and nanoribbon volumes are $V=A_{B} C$, where $A_{B}$ is the 


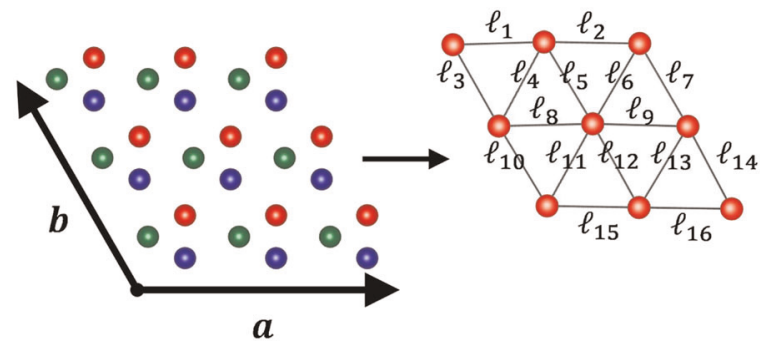

Fig. 10 Illustration of the calculation methodology of a for the $\boldsymbol{N}=\mathbf{3}$ rhomboid nanowire. All green, blue, and red atoms are in separate planes. There are 16 distances to average to calculate $\tilde{\boldsymbol{a}}$ for the $N=3$ rhomboid nanowire example.

base area and $c$ is the lattice constant in the $z$-direction. We use a hexagon $\left(A_{\mathrm{B}}=3 \sqrt{3}(N \tilde{a})^{2} / 2\right)$, a rhombus $\left(A_{\mathrm{B}}=\sqrt{3}(N \tilde{a})^{2} / 2\right)$, and an equilateral triangle $\left(A_{\mathrm{B}}=\sqrt{3}(N \tilde{a})^{2} / 4\right)$ for the nanowire base areas. We use parallelograms $\left(A_{\mathrm{B}}=L R \tilde{a}_{\mathrm{L}}^{2} / 2\right)$ for the nanoribbon base areas, where the quasi-lattice constants for the nanoribbons $\tilde{a}_{\perp}$ are taken to be the quasilattice constant of the rhomboid nanowires of the same size. No surface-tovolume ratio is computed for the monolayer.

Lateral surface areas for hexagonal, rhomboid, and triangular t-Te nanowire unit cells consist of six $\left(N_{R}=6\right)$, four $\left(N_{R}=4\right)$, and three $\left(N_{R}=3\right)$ rectangular side walls; the side length along the axial direction is $c$, and the side lengths along the transverse direction (other directions) are integer multiples of the quasi-lattice constant $\tilde{a}$, yielding a total lateral surface area $A_{L}=N_{R} N a \tilde{c}$, where $N_{\mathrm{R}}$ is number of side walls and $N$ is number of Te-helices per side of a t-Te nanowire. The lateral surface area for the nanoribbons are computed in the same manner with rectangular side walls, yielding a total lateral surface area $A_{\mathrm{L}}=2 N_{\mathrm{L}} \tilde{a}_{\mathrm{L}} \mathrm{C}+2 R \tilde{a}_{\mathrm{L}} \mathrm{C}$, where $N_{\mathrm{L}}$ is the number of layers.

\section{Formation energy per Te-helix}

To gain a deeper understanding of the formation energy of the nanostructures under study, we decompose the formation energy of a nanostructure $E_{\mathrm{F}}$ into a sum of energy penalties $\epsilon_{m}$ associated with each composing helix. More precisely, we assume the formation energy of a t-Te nanostructure to be:

$$
E_{\mathrm{F}} \approx \sum_{m=1}^{6} \frac{n_{m} \epsilon_{m}}{N_{\mathrm{tot}}}
$$

where $n_{m}$ is the number of Te-helices with $m$ missing helical neighbors, while $\epsilon_{m}$ is its energy penalty (per supercell). $N_{\text {tot }}$ is the number of atoms per supercell.

Determining $\epsilon_{6}$ is trivial. It is defined as the total energy difference between a single Te-helix and a helix in bulk t-Te. To determine $\epsilon_{m}$ for $m<$ 6 , we perform an OLS fit on Eq. (2), for a range of reference structures. We include 40 1D structures (nanowires and nanoribbons) in the OLS fit. We exclude the monolayer nanoribbon (Fig. 3d) structures from our reference because the monolayer material forms in a different stable phase, with covalent bonds rather than vdW-interacting helices. None of our reference structures have helices with one or five helical neighbors. Therefore, we only provide results for $m \in(0,2,3,4,6)$. The root-mean square error of our formation energy with the $\epsilon_{m}$ value is $1.9 \mathrm{meV} /$ atom.

In summary, our calculations show that hexagonal- or triangular-shaped $\mathrm{t}$-Te nanowires rather than layers of Te will preferentially form. The relative stability of nanowires is caused by a higher saturation of the vdW interactions between Te-helices. Based on the number of neighbors of each Te-helix, we obtain simple models that describe the formation energy and bandgap of any nanostructure of t-Te. Larger t-Te nanowires $\left(>6 \mathrm{~nm}^{2}\right)$ have indirect or nearly direct bandgaps measuring around $0.8 \mathrm{eV}$. Smaller $\mathrm{t}$-Te nanowires $\left(<4 \mathrm{~nm}^{2}\right)$ feature indirect bandgaps exceeding $1 \mathrm{eV}$.

The increase in bandgap in nanowires, compared to bulk at extremely scaled dimensions, makes it apparent that the large leakage currents observed in recent Te transistor prototypes is not inevitable. Furthermore, since t-Te consists of helices with vdW bonds in between them, nanowires are naturally terminated compared to bulk or $2 \mathrm{D}$ materials that need specific surface terminations to avoid interface/edge states. Since t-Te is the only material that combines a low anticipated off-current, the ability to grow at low temperature, a prospect of high mobility, with naturally terminated surfaces, $\mathrm{t}-\mathrm{Te}$ is a legitimate contender to succeed $\mathrm{Si}$ in the realm of extremely scaled nanowire FETs.

\section{DATA AVAILABILITY}

The data that support the findings of this study are available from the corresponding author upon request.

\section{CODE AVAILABILITY}

All data were generated using the VASP, and no custom computer code was employed.

Received: 20 December 2019; Accepted: 1 April 2020; Published online: 18 May 2020

\section{REFERENCES}

1. Gamiz, F. \& Fischetti, M. V. Monte Carlo simulation of double-gate silicon-oninsulator inversion layers: the role of volume inversion. J. Appl. Phys. 89, 5478-5487 (2001).

2. Fischetti, M. V., Fu, B. \& Vandenberghe, W. G. Theoretical study of the gate leakage current in sub-10-nm field-effect transistors. IEEE Trans. Electron Dev. 60, 3862-3869 (2013).

3. Neto, A. H. C., Guinea, F., Pres, N. M. R., Nooselov, K. S. \& Geim, A. K. The electronic properties of graphene. Rev. Mod. Phys. 81, 109-162 (2009).

4. Tiwari, S., Van de Put, M. L., Sorrée, B. \& Vandenberghe, W. G. Carrier transport in two-dimensional topological insulator nanoribbons in the presence of vacancy defects. 2D Mater. 6, 025011-025024 (2019).

5. Vandenberghe, W. G. \& Fishetti, M. V. Imperfect two-dimensional topological insulator field-effect transistors. Nat. Commun. 8, 14184-14191 (2017).

6. Khatami, M. M. et al. Electronic transport properties of silicane determined from first principles. Materials 12, 2935-2950 (2019).

7. Manzeli, S., Ovchinnikov, D., Pasquier, D., Yazyev, O. V. \& Kis, A. 2D transition metal dichalcogenides. Nat. Rev. Mater. 2, 17033-17048 (2017).

8. Laturia, A., Van de Put, M. L. \& Vandenberghe, W. G. Dielectric properties of hexagonal boron nitride and transition metal dichalcogenides: from monolayer to bulk. npj 2D Mater. Appl. 2, 6-11 (2018).

9. Gaddemane, G. et al. Theoretical studies of electronic transport in monolayer and bilayer phosphorene: a critical overview. Phys. Rev. B 98, 115416-115432 (2018).

10. Zhou, G. et al. High-mobility helical Tellurium field-effect transistors enabled by transfer-free, low-temperature direct growth. Adv. Mater. 30, 1803109-1803116 (2018).

11. Wang, $Y$. et al. Field-effect transistors made from solution-grown two-dimensional tellurene. Nat. Electron. 1, 228-236 (2018).

12. Anzin, V. B., Eremets, M. I., Kosichkin, Yu. V., Nadezhdinskii, A. I. \& Shirokov, A. M. Measurement of the energy gap in Tellurium under pressure. Phys. Stat. Sol. 42, 385-390 (1977).

13. Peng, H., Kioussis, N. \& Snyder, G. J. Elemental tellurium as a chiral p-type thermoelectric material. Phys. Rev. B 89, 195206-195213 (2014).

14. Lee, T. I. et al. High-power density piezoelectric energy harvesting using radially strained ultrathin trigonal tellurium nanowire assembly. Adv. Mater. 25, 2920-2925 (2013).

15. $\mathrm{Xu}, \mathrm{W}$. et al. Structural, electrical, and photoconductive properties of individual single-crystalline tellurium nanotubes synthesized by a chemical route: doping effects on electrical structure. Small 4, 888-893 (2008).

16. Zogg, $\mathrm{H}$. et al. Photovoltaic infrared sensor arrays in monolithic lead chalcogenides on silicon. Sci. Technol. 6, C36-C41 (1991).

17. Qin, J. et al. Raman response and transport properties of tellurium atomic chains encapsulated in nanotubes. Nat. Electron. 3, 141-147 (2020).

18. Joannopoulos, J. D., Schluter, M. \& Cohen, M. L. Electronic structure of trigonal and amorphous Se and Te. Phys. Rev. B 11, 2186-2199 (1975).

19. Novoselov, K. S. et al. Two-dimensional gas of massless Dirac fermions in graphene. Nature 438, 197-200 (2005)

20. Geim, A. K. \& Novoselov, K. S. The rise of graphene. Nat. Mater. 6, 183 (2007).

21. Moors, K. et al. Theoretical study of scattering in graphene ribbons in the presence of structural and atomistic edge roughness. Phys. Rev. Mater. 3, 024001-024012 (2019).

22. Joensen, P., Frindt, R. F. \& Morrison, S. R. Single layer MoS2. Mater. Res. Bull. 21, 457-461 (1986). 
23. Zhu, Z. et al. Multivalency-driven formation of Te-based monolayer materials: a combined first-principles and experimental study. Phys. Rev. Lett. 119, 106101-106106 (2017).

24. Liu, D., Lin, X. \& Tomanek, D. Microscopic mechanism of the helix-to-layer transformation in elemental group VI solids. Nano Lett. 18, 4908-4913 (2018).

25. Xian, L., Pax, A. P., Bianco, E., Ajayan, P. M. \& Rubio, A. Square selenene and tellurene: novel group $\mathrm{VI}$ elemental $2 \mathrm{D}$ materials with nontrivial topological properties. 2D Mater. 4, 041003-041010 (2017).

26. Huang, X. et al. Epitaxial growth and band structure of Te film on graphene. Nano Lett. 8, 4619-4623 (2017).

27. Asendorf, R. H. Space group of tellurium and selenium. J. Chem. Phys. 27, 11-16 (1957).

28. Bradley, A. J. The crystal structures of the rhombohedral forms of selenium and tellurium. Philos. Mag. 48, 477-496 (1924).

29. Cherin, P. U. P. Two-dimensional refinement of the crystal structure of tellurium. Acta Crystallogr. 23, 670-671 (1967).

30. Furukawa, T., Shimokawa, Y., Kobayashi, K., Shimokawa. \& Tetsuaki, I. Observation of current-induced bulk magnetization in elemental tellurium. Nat. Commun. 8, 954-959 (2017).

31. Yan, J. et al. Monolayer tellurene-metal contacts. J. Mater. Chem. C 6, 6153-6163 (2018).

32. Sharma, S., Singh, N. \& Schwingenschlogl, U. Two-dimensional tellurene as excellent thermoelectric material. ACS Appl. Energy Mater. 1, 1950-1954 (2018).

33. Qiao, J. et al. Few-layer Tellurium: one-dimensional-like layered elementary semiconductor with striking physical properties. Sci. Bull. 63, 159-168 (2018).

34. Yi, S., Zhu, Z., Cai, X., Jia, Y. \& Cho, J. The nature of bonding in bulk tellurium composed of one-dimensional helical chains. Inorg. Chem. 57, 5083-5088 (2018).

35. Keller, R., Holzapfel, W. B. \& Schulz, H. Effect of pressure on the atom positions in Se and Te. Phys. Rev. B. 15, 4404-4412 (1977).

36. Vandenberghe, W. G. \& Fischetti, M. V. Advanced Physics of Electron Transport in Semiconductors and Nanostructures 102-110 (Springer International Publishing Switzerland, 2016).

37. Li, Y., Sun, Y., Na, Y., Saidi, W. A. \& Zhang, L. Diverse electronic properties of 2D layered Se-containing materials composed of quasi-1D atomic chains. Phys. Chem. Chem. Phys. 22, 2122-2129 (2020).

38. Mayers, B. \& Xia, Y. One-dimensional nanostructures of trigonal tellurium with various morphologies can be synthesized using a solution-phase approach. $J$. Mater. Chem. 12, 1875-1881 (2002).

39. Joubert, D. \& Kresse, G. From ultrasoft pseudopotentials to the projector augmented-wave method. Phys. Rev. B 59, 1758-1775 (1999).

40. Perdew, J. P., Burke, K. \& Ernzerhof, M. Generalized gradient approximation made simple. Phys. Rev. Lett. 77, 3865-3868 (1996).

41. Grimme, S., Antony, J., Ehrlich, S. \& Krieg, S. A consistent and accurate ab initio parametrization of density functional dispersion correct (DFT-D). J. Chem. Phys. 132, 154104-154123 (2010).

42. Monkhorst, H. J. \& Pack, J. D. Special points for Brillouin-zone integrations. Phys. Rev. B 13, 5188-5192 (1976).

43. Becke, A. D. Density-functional exchange-energy approximation with correct asymptotic behavior. Phys. Rev. A 38, 3098-3100 (1988).
44. Heyd, J., Scuseria, G. E. \& Ernzerhof, Z. Erratum: "Hybrid functionals based on a screened Coulomb potential" [J. Chem. Phys. 118, 8207 (2003)]. J. Chem. Phys. 124, 219906 (2006).

45. Steiner, S., Khmelevskyi, S., Marsmann, M. \& Kresse, G. Calculation of the magnetic anisotropy with projected-augmented-wave methodology and the case study of disordered Fe1-x Cox alloys. Phys. Rev. B 93, 22425-22431 (2016).

\section{ACKNOWLEDGEMENTS}

We acknowledge the Texas Advanced Computing Center (TACC) at The University of Texas at Austin for providing the high-performance computing resources that have contributed to the research results reported within this paper. URL: http://www.tacc. utexas.edu.

\section{AUTHOR CONTRIBUTIONS}

W.G.V. and C.L.H. conceived the project. A.K. performed the simulations and the results obtained were analyzed by A.K., M.L.V.de.P. and W.G.V. A.K. wrote the paper with all the authors contributing to the discussion and preparation of the manuscript.

\section{COMPETING INTERESTS}

The authors declare no competing interests.

\section{ADDITIONAL INFORMATION}

Correspondence and requests for materials should be addressed to W.G.V.

Reprints and permission information is available at http://www.nature.com/ reprints

Publisher's note Springer Nature remains neutral with regard to jurisdictional claims in published maps and institutional affiliations.

(i) Open Access This article is licensed under a Creative Commons Attribution 4.0 International License, which permits use, sharing, adaptation, distribution and reproduction in any medium or format, as long as you give appropriate credit to the original author(s) and the source, provide a link to the Creative Commons license, and indicate if changes were made. The images or other third party material in this article are included in the article's Creative Commons license, unless indicated otherwise in a credit line to the material. If material is not included in the article's Creative Commons license and your intended use is not permitted by statutory regulation or exceeds the permitted use, you will need to obtain permission directly from the copyright holder. To view a copy of this license, visit http://creativecommons. org/licenses/by/4.0/

(c) The Author(s) 2020 